\title{
Identifying Missed Opportunities to Curtail Antimicrobial Therapy for Presumed Ventilator-Associated Pneumonia Using the Clinical Pulmonary Infection Score
}

\author{
Sean K Gorman, Lynne-Michelle M Stewart, Richard S Slavik, Jane de Lemos, Dean Chittock, \\ Vinay K Dhingra, Juan J Ronco, and Harjinder Parwana
}

\begin{abstract}
Background: Early discontinuation of antimicrobial therapy for ventilator-associated pneumonia can reduce the emergence of antimicrobial resistance, the occurrence of adverse drug events, and the cost of therapy. Evidence suggests that discontinuation of therapy by day 3 may be appropriate for patients with a clinical pulmonary infection score of 6 or less at baseline and on day 3 .
\end{abstract}

Objectives: To determine the proportion of patients eligible for antimicrobial discontinuation on day 3 and day 7 of therapy and to determine the proportion of eligible patients for whom antimicrobials were discontinued within these timeframes.

Methods: A 6-month observational study was conducted from October 3, 2005, to March 31, 2006, in a 27-bed medical-surgical tertiary care intensive care unit. Clinical pharmacists attended daily rounds and prospectively identified patients for inclusion in the study. A study pharmacist retrospectively calculated clinical pulmonary infection scores. Other data were obtained from the quality-improvement database and patient health records for the intensive care unit.

Results: Ninety-two patients were treated for ventilator-associated pneumonia during the study period, of whom 49 were included in the analysis. At day 3, 17 (35\%) of the 49 patients were eligible for early discontinuation of antimicrobial therapy, but therapy was discontinued for only $2(12 \%)$ of these 17 patients. At day 7, 10 (32\%) of 31 patients were eligible for antimicrobial discontinuation, but therapy was discontinued for only $1(10 \%)$ of these 10 patients.

Conclusions: A significant opportunity exists at the authors' institution to develop and implement an antimicrobial discontinuation policy that uses the clinical pulmonary infection score to guide antimicrobial use for patients with ventilator-associated pneumonia.

Key words: ventilator-associated pneumonia, artificial respiration, clinical pulmonary infection score, antibacterial agents

Can J Hosp Pharm 2009;62(3):217-225

\section{RÉSUMÉ}

Contexte : Larrêt précoce de l'antibiothérapie dans les cas de pneumonie sous ventilation assistée pourrait réduire l'apparition de résistance antimocrobienne, la survenue d'événements indésirables liés aux médicaments et le coût du traitement. Des données suggèrent que l'arrêt du traitement au jour 3 pourrait être approprié chez les patients présentant un score d'infection pulmonaire clinique de 6 ou moins au début et au $3^{\mathrm{e}}$ jour du traitement.

Objectifs : Déterminer la proportion de patients admissibles à l'arrêt de l'antibiothérapie, aux jours 3 et 7 du traitement, et la proportion de ces patients dont l'antibiothérapie a été effectivement interrompue à ces dates.

Méthodes : Une étude d'observation de six mois a été menée du 3 octobre 2005 au 31 mars 2006, dans une unité de soins intensifs médico-chirurgicale de 27 lits d'un hôpital de soins tertiaires. Des pharmaciens cliniciens participaient aux tournées quotidiennes et ont répertorié de façon prospective les patients admissibles à l'étude. Un pharmacien investigateur a calculé de façon rétrospective les scores d'infection pulmonaire clinique. D'autres données ont aussi été collectées de la base de données sur l'amélioration de la qualité et des dossiers médicaux des patients de l'unité de soins intensifs.

Résultats : En tout, 92 patients ont reçu une antibiothérapie pour une pneumonie sous ventilation assistée au cours de la période de l'étude, et 49 d'entre eux ont été retenus aux fins d'analyse. Au jour 3, 17 (35\%) des 49 patients étaient admissibles à un arrêt précoce de l'antibiothérapie, mais on a procédé à l'arrêt de l'antibiothérapie chez seulement 2 (12\%) de ces 17 patients. Au jour 7, 10 patients (32\%) sur 31 étaient admissibles à l'arrêt de l'antibiothérapie, mais on a interrompu l'antibiothérapie chez seulement $1(10 \%)$ de ces 10 patients.

Conclusions : L'établissement des auteurs se prêterait particulièrement bien à l'élaboration et la mise en place d'une politique d'arrêt de l'antibiothérapie, qui se fonderait sur les scores d'infection pulmonaire clinique dans les cas de pneumonie sous ventilation assistée.

Mots clés : pneumonie sous ventilation assistée, respiration artificielle, score d'infection pulmonaire clinique, antibiotiques

[Traduction par l'éditeur] 


\section{INTRODUCTION}

$\mathrm{V}$ entilator-associated pneumonia, or pneumonia that arises more than $48-72 \mathrm{~h}$ after endotracheal intubation, is a significant complication for mechanically ventilated patients. ${ }^{1}$ Of all intubated patients, $8 \%$ to $28 \%$ will experience this complication, which makes it the second most common form of nosocomial infection and the most common form of infection afflicting intubated patients. ${ }^{2-4}$ Lower respiratory tract infections account for $49 \%$ of all antimicrobials prescribed in the intensive care unit (ICU). ${ }^{5}$ However, in one study, 63\% of the antimicrobials used were for respiratory tract infections that were clinically suspected, rather than proven. ${ }^{6}$ In an attempt to improve the specificity of clinical diagnosis of ventilatorassociated pneumonia, Pugin and others ${ }^{7}$ created the clinical pulmonary infection score (CPIS), which combines the weighted scores of clinical, radiographic, physiologic, and microbiologic variables into one numeric value. A CPIS greater than 6 has been found to correlate with the presence of pneumonia. ${ }^{7}$

Although early, appropriate antimicrobial therapy in adequate doses is critical for the management of ventilatorassociated pneumonia, it is also important to frequently reassess the need for antimicrobials and to rationalize the duration of therapy to the minimum effective period. This approach is intended to avoid adverse drug events, prevent the development of antimicrobial resistance, and reduce costs. ${ }^{1,8}$ The 2005 American Thoracic Society guidelines for the management of ventilator-associated pneumonia in adults recommended 7 to 8 days of antimicrobial therapy for patients with uncomplicated pneumonia who have received appropriate initial antimicrobial therapy with a good clinical response and who have no evidence of infection with Pseudomonas aeruginosa. ${ }^{1}$ It is recommended that patients with suspected or proven ventilator-associated pneumonia due to $P$. aeruginosa receive longer-duration therapy, such as 14 to 21 days, ${ }^{1,8}$ but it may be possible to shorten antimicrobial therapy for this condition to less than 7 or 8 days. In one randomized controlled trial, a formal antimicrobial discontinuation policy helped to reduce the duration of antimicrobial therapy from 8 days to 6 days. ${ }^{9}$ Although that trial did not use the CPIS to guide the decision to discontinue antimicrobial therapy, it did use components of the CPIS. Data from another randomized controlled trial showed that for patients with hospital-acquired pneumonia or ventilator-associated pneumonia who had a CPIS of up to 6 at both day 0 and day 3 of therapy, antimicrobial therapy could be discontinued at day 3 with no increase in mortality relative to patients who received a longer course of therapy. ${ }^{6}$ Furthermore, patients who received short-course therapy had a shorter length of stay in the ICU, lower costs, less antimicrobial resistance, and fewer superinfections relative to patients who received standard-course therapy. ${ }^{6}$
The Vancouver General Hospital currently has no formal strategy to guide discontinuation of antimicrobials for ventilator-associated pneumonia, but it is not known if such a strategy is even required, given the lack of information on antimicrobial use for this condition at this institution. Therefore, the aim of this study was to determine the proportion of patients with ventilatorassociated pneumonia at the Vancouver General Hospital who would be eligible for short-course antimicrobial therapy. By determining whether antimicrobials were discontinued for these patients by day 3 and by day 7 of therapy, the need for a CPIS-guided antimicrobial discontinuation policy can be identified.

\section{METHODS}

\section{Study Design and Organization}

This retrospective study was conducted in the 27-bed medical-surgical ICU of a tertiary care referral teaching centre from October 3, 2005, to March 31, 2006. The Clinical Research Ethics Board at the University of British Columbia and the Vancouver Coastal Health Research Institute approved the study protocol.

\section{Patients}

Patients were eligible for inclusion in the study if they were admitted to the ICU during the study period, were at least 18 years of age, and were receiving antimicrobial therapy for presumed ventilator-associated pneumonia. For the purposes of this study, patients who were receiving antimicrobials for presumed pneumonia that had developed more than $48 \mathrm{~h}$ after intubation were deemed to have ventilator-associated pneumonia.

Patients were excluded if their antimicrobial therapy for ventilator-associated pneumonia had been initiated outside of the ICU, if they had received antimicrobial therapy for prior ventilator-associated pneumonia within the previous $48 \mathrm{~h}$, if they had a concomitant infection requiring the same antimicrobials as would be used for ventilator-associated pneumonia, if they had induced hypothermia or immunosuppression (due to HIV infection, hematologic deficiency, neutropenia induced by cytotoxic chemotherapy, or solid organ transplantation), or if they were discharged from the ICU or died within $72 \mathrm{~h}$ after starting antimicrobial therapy for ventilator-associated pneumonia.

\section{Antimicrobial Treatments}

The selection and duration of antimicrobial therapy was at the discretion of the ICU clinicians, and the duration of therapy was not guided by use of the CPIS. 


\section{Data Collection, Baseline Assessment, and Follow-up}

Clinical pharmacists who attended daily interdisciplinary patient care rounds in the ICU on weekdays (Monday to Friday inclusive) prospectively identified new cases of presumed ventilator-associated pneumonia and reviewed patients' health care records every Monday to identify new cases diagnosed over the weekend. The clinical pharmacists' role was to perform routine daily activities related to the provision of pharmaceutical care. This included documenting the ICU physician's interpretation of the chest radiograph. Interpretation of chest radiograph data (specifically, whether there was no infiltrate,

\section{Table 1. Calculation of the Clinical Pulmonary Infection Score (CPIS)*}

\begin{tabular}{|c|c|c|}
\hline Variable no. & Variable & Pointst \\
\hline \multirow[t]{4}{*}{1} & Temperature $\left({ }^{\circ} \mathrm{C}\right)$ & \\
\hline & $\geq 36.5$ and $\leq 38.4$ & 0 \\
\hline & $\geq 38.5$ and $\leq 38.9$ & 1 \\
\hline & $\geq 39$ or $\leq 36$ & 2 \\
\hline \multirow[t]{4}{*}{2} & Blood leukocytes (× 109/L) & \\
\hline & $\geq 4$ and $\leq 11$ & 0 \\
\hline & $<4$ or $>11$ & 1 \\
\hline & $<4$ or $>11+$ band forms $\geq 50 \%$ & 2 \\
\hline \multirow[t]{4}{*}{3} & Tracheal secretions & \\
\hline & Absent & 0 \\
\hline & Present, nonpurulent & 1 \\
\hline & Present, purulent & 2 \\
\hline \multirow[t]{3}{*}{4} & Oxygenation $\left(\mathrm{PaO}_{2} / \mathrm{FiO}_{2}, \mathrm{~mm} \mathrm{Hg}\right)$ & \\
\hline & $>240$ or ARDS & 0 \\
\hline & $\leq 240$ and no ARDS & 2 \\
\hline \multirow[t]{4}{*}{5} & Pulmonary radiography & \\
\hline & No infiltrate & 0 \\
\hline & Diffuse (or patchy) infiltrate & 1 \\
\hline & Localized infiltrate & 2 \\
\hline \multirow[t]{3}{*}{6} & Progression of pulmonary infiltrate & \\
\hline & No radiographic progression & 0 \\
\hline & $\begin{array}{l}\text { Radiographic progression } \\
\text { (after exclusion of CHF and ARDS) }\end{array}$ & 2 \\
\hline \multirow[t]{5}{*}{7} & Culture of tracheal aspirate & \\
\hline & No growth of pathogenic bacteriał & 0 \\
\hline & Pathogenic bacteria cultured & 1 \\
\hline & $\begin{array}{l}\text { Pathogenic bacteria cultured + } \\
\text { same bacteria as seen with }\end{array}$ & \\
\hline & Gram staining & 2 \\
\hline
\end{tabular}

ARDS = acute respiratory distress syndrome, $\mathrm{CHF}=$ congestive heart failure, $\mathrm{PaO}_{2} / \mathrm{FiO}_{2}=$ arterial oxygen pressure divided by fraction of inspired oxygen.

*Adapted, with permission, from Singh $\mathrm{N}$ et al. Short-course empiric antibiotic therapy for patients with pulmonary infiltrates in the intensive care unit: a proposed solution for indiscriminate antibiotic prescription. Am J Respir Crit Care Med 2000;162 (2 Pt 1):505-511. (C) American Thoracic Society. Based on the original designed by Pugin et al. ${ }^{7}$

tPoints are summed to determine the CPIS. For baseline CPIS, the score was calculated from variables 1 through 5 (maximum score 10). For CPIS at day 3 and day 7, the score was calculated from variables 1 through 7 (maximum score 14).

$\neq$ As the predominant organism in the culture. diffuse or patchy infiltrate, or localized infiltrate) was obtained from the ICU physician on day 0 , day 3 , and day 7 of antimicrobial therapy. In addition, information about radiographic progression of an infiltrate was obtained on day 3 and day 7 . In cases where the ICU physician's interpretation was not available, the radiologist's dictated interpretation was obtained from the Patient Care Information System. The clinical pharmacists did not calculate the CPIS for any of the patients identified for potential inclusion in the study.

Upon review of the patient's health care record and data in the Patient Care Information System, the study pharmacist (L.M.S.) recorded the presence of inclusion or exclusion criteria, the patient's age and sex, the reason or reasons for mechanical ventilation (mixed respiratory failure; trauma; neurological or neuromuscular disease; postoperative respiratory failure; cardiogenic pulmonary edema; acute lung injury or acute respiratory distress syndrome; gastrointestinal bleeding; asthma, chronic obstructive pulmonary disease, or acute exacerbation of chronic bronchitis), whether traditional criteria for ventilator-associated pneumonia were met (defined as having been intubated for $48 \mathrm{~h}$ or more, having new or progressive infiltrate on chest radiography, and having 2 of the following: hyperthermia, leukopenia or leukocytosis, and presence of purulent secretions), duration of ICU stay, death in the ICU, early or late ventilator-associated pneumonia (with early being defined as occurring within the first 4 days of mechanical ventilation), number of episodes of ventilatorassociated pneumonia, results of Gram staining and culture at day 0 , initial antimicrobial therapy, and duration of antimicrobial therapy. At day 0 , day 3, and day 7 of therapy, use of vasopressor (norepinephrine, dopamine, epinephrine, phenylephrine, or vasopressin), presence of a concomitant infection, and CPIS (Table 1) were recorded. ${ }^{6,7}$ The 2 components of the CPIS that are somewhat subjective are the interpretation of the chest radiograph and the quality of tracheal secretions. Collection and interpretation of chest radiograph data have been described above. The tracheal secretion score was obtained from the respiratory section of the daily critical care flow sheet. The registered respiratory therapist or registered nurse described the quality of the secretions as mucoid, mucopurulent, purulent, bloody, or mucobloody. For the purposes of calculating the CPIS, 2 points were scored for mucopurulent or purulent secretions. The study pharmacist (L.M.S.) who collected all data and calculated all CPIS values was not involved in patient care. The results of the CPIS were not shared with the health care team, including the ICU clinical pharmacists. Acute Physiology and Chronic Health Evaluation II (APACHE II) scores were obtained at the time of ICU admission using the quality assurance database of the Vancouver General Hospital ICU. 


\section{Outcome Measures}

The primary outcome was the proportion of patients treated for ventilator-associated pneumonia who could have had antimicrobial therapy discontinued after 3 days of therapy, as indicated by CPIS $\leq 6$ at day 0 and day 3 of therapy.

The secondary outcomes were the proportion of patients treated for ventilator-associated pneumonia who could have had antimicrobial therapy discontinued after 7 days of therapy, as indicated by CPIS $\leq 6$ at day 0 and day 7 of therapy; the proportion who had CPIS $\leq 6$ or less at day 0 and day 3 for whom antimicrobial therapy continued for no more than 3 days; and the proportion who had CPIS $\leq 6$ at day 0 and day 7 of therapy for whom antimicrobials were continued for no more than 7 days.

\section{Data Management and Statistical Analysis}

Data were entered into a Microsoft Excel (Microsoft, Redmond, Washington) database. Standard descriptive statistics are reported, including means and standard deviations for normally distributed data.

\section{RESULTS}

\section{Clinical Characteristics}

A total of 466 patients were admitted to the ICU between October 3, 2005, and March 31, 2006. Ninety-two (20\%) patients were identified as being treated for ventilatorassociated pneumonia during the ICU stay, and $43(47 \%)$ of those patients were excluded (Figure 1). The clinical characteristics of the 49 patients who were included in the study are listed in Table 2 . The sample was primarily male (67\%), with a mean age of $54 \pm 19$ years. Mixed respiratory failure was the predominant reason for mechanical ventilation (33\%). The mean APACHE II score on ICU admission was $20 \pm 6$, and the length of the ICU stay was $22 \pm 14$ days. Four patients $(8 \%)$ died while in the ICU. Vasopressors were administered to $8(16 \%)$ of the patients on day 0 of antimicrobial therapy for ventilator-associated pneumonia. Concomitant infection was present in $16(33 \%)$ patients on day 0 ; however, these infections required antimicrobials with a different spectrum of activity than those required for treatment of ventilator-associated pneumonia. Thirty $(61 \%)$ patients met the traditional criteria for ventilatorassociated pneumonia on day 0 . Early ventilator-associated pneumonia was present in $21(43 \%)$ patients, and $3(6 \%)$ patients experienced more than 1 episode of the condition. Initial antimicrobial therapy for ventilator-associated pneumonia was deemed appropriate in $26(96 \%)$ of the 27 patients who had positive results on sputum culture. Antimicrobials were administered for an average of $15 \pm 11$ days.

\section{Microbiologic Characteristics}

The results of Gram staining were positive in 35 (71\%) patients, but culture of sputum yielded pathogenic organisms for only $28(57 \%)$ of the patients (Table 3). Methicillinsensitive Staphylococcus aureus was isolated from 10 (20\%) patients and Klebsiella pneumoniae from 5 (10\%) patients. Non-lactose-fermenting gram-negative bacilli were isolated from $5(10 \%)$ patients or $18 \%$ of all positive sputum cultures. Methicillin-resistant Staphylococcus aureus was isolated in $1(2 \%)$ patient or $4 \%$ of all positive sputum cultures.

\section{Antimicrobial Characteristics}

Ticarcillin-clavulanate was the initial antimicrobial prescribed for 22 (45\%) patients (Table 4). A total of 26 patients (53\%) received an antimicrobial regimen containing ticarcillin-clavulanate. Ciprofloxacin was the initial antimicrobial prescribed for 7 (14\%) patients, and a total of $14(29 \%)$ received a regimen containing ciprofloxacin. A vancomycincontaining regimen was administered to $8(16 \%)$ patients, and cefuroxime was administered to $5(10 \%)$ patients.

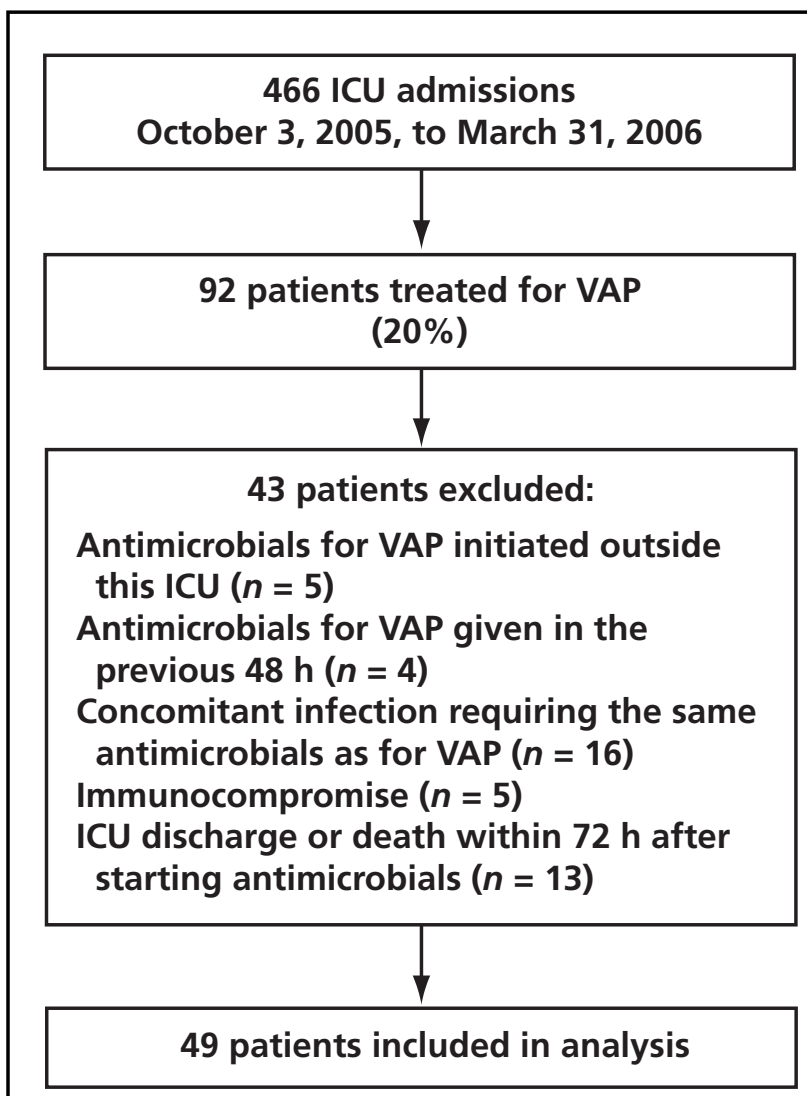

Figure 1. Trial flow diagram. ICU = intensive care unit, $\mathrm{VAP}=$ ventilator-associated pneumonia 
Table 2. Clinical Characteristics of Patients

\begin{tabular}{|c|c|c|}
\hline \multirow{3}{*}{$\begin{array}{l}\text { Characteristic } \\
\text { Age, yr (mean } \pm \text { SD) } \\
\text { Sex (no. of males) }\end{array}$} & \multicolumn{2}{|c|}{ No. $(\%)$ of Patients* $(n=49)$} \\
\hline & \multicolumn{2}{|c|}{$54 \pm 19$} \\
\hline & 33 & $(67)$ \\
\hline \multicolumn{3}{|l|}{ Reason for mechanical ventilationt } \\
\hline Mixed respiratory failure & 16 & (33) \\
\hline Trauma & 14 & (29) \\
\hline Neurological or neuromuscular disease & 13 & $(27)$ \\
\hline Postoperative respiratory failure & 6 & $(12)$ \\
\hline Cardiogenic pulmonary edema & 3 & (6) \\
\hline Acute lung injury or ARDS & 3 & (6) \\
\hline Gl bleeding & 2 & (4) \\
\hline Asthma, COPD, or AECB & 1 & $(2)$ \\
\hline \multicolumn{3}{|l|}{ Data related to ICU stay } \\
\hline APACHE II on ICU admission (mean \pm SD) & \\
\hline Duration of ICU stay, days (mean \pm SD) & \multicolumn{2}{|c|}{$22 \pm 14$} \\
\hline Death in the ICU & 4 & (8) \\
\hline \multicolumn{3}{|l|}{ Vasopressor use } \\
\hline Day 0 & 8 & $(16)$ \\
\hline Day 3 & 5 & $(10)$ \\
\hline Day 7 & 2 & (4) \\
\hline \multicolumn{3}{|l|}{ Concomitant infection } \\
\hline Day 0 & 16 & (33) \\
\hline Day 3 & 17 & (35) \\
\hline Day 7 & 23 & (47) \\
\hline \multicolumn{3}{|l|}{ Data related to diagnosis of ventilator-associated pneumonia } \\
\hline Met traditional criteria on day $0 \neq$ & 30 & (61) \\
\hline Early§ & 21 & (43) \\
\hline$>1$ episode & 3 & (6) \\
\hline Appropriate initial antimicrobial therapy $(n=27)$ & 26 & (96) \\
\hline Duration of antimicrobial therapy, days (mean $\pm \mathrm{SD}$ ) & \multicolumn{2}{|c|}{$15 \pm 11$} \\
\hline \multicolumn{3}{|c|}{ 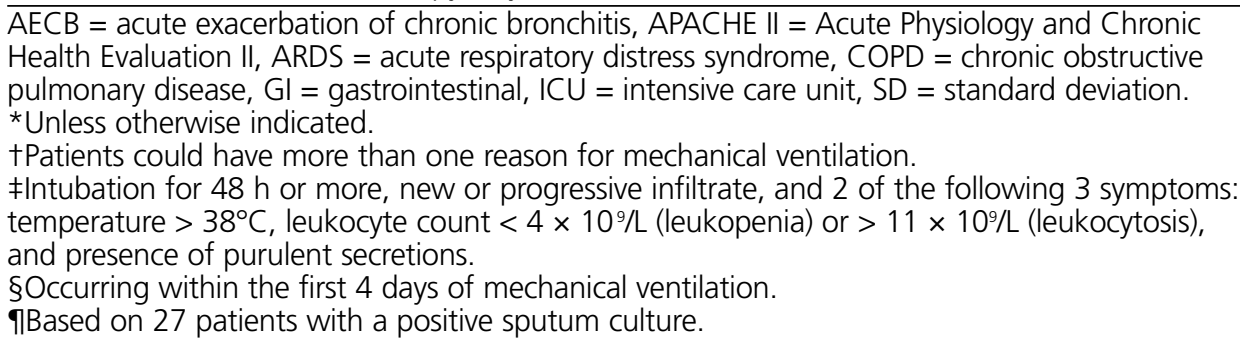 } \\
\hline
\end{tabular}

\section{Primary Outcome}

A total of 49 patients were eligible for assessment at day 3 of antimicrobial therapy. For 17 (35\%) of these, antimicrobials could have been discontinued at that point, as indicated by CPIS $\leq 6$ on both day 0 and day 3 of therapy (Figure 2).

\section{Secondary Outcomes}

By day 7 of antimicrobial therapy, 31 patients were eligible for assessment. For 10 (32\%) of these, antimicrobials could have been discontinued, as indicated by CPIS $\leq 6$ at both day 0 and day 7 of therapy (Figure 3). By day 3 of therapy, antimicrobial therapy had been discontinued for 2 (12\%) of the eligible patients (Figure 2). By day 7 of therapy, antimicrobial therapy had been discontinued for $1(10 \%)$ eligible patient (Figure 3).

\section{DISCUSSION}

This small observational study from a 27-bed tertiary care ICU has revealed a significant need for a systematic policy for discontinuation of antimicrobials. More than one-third of the patients included in the analysis were eligible for a short course of antimicrobial therapy for ventilator-associated pneumonia, but fewer than $15 \%$ of these patients had antimicrobial therapy curtailed by day 3 of therapy. This suggests that improvements can be made in rationalizing the duration of 
Table 3. Results of Sputum Culture

\begin{tabular}{|c|c|c|}
\hline Microorganism & & $\begin{array}{l}\text { f Patients } \\
\text { 49) }\end{array}$ \\
\hline Positive result on Gram staining & 35 & (71) \\
\hline Positive result on sputum culture* & 28 & $(57)$ \\
\hline Methicillin-sensitive Staphylococcus aureus & 10 & $(20)$ \\
\hline Klebsiella pneumonia & 5 & (10) \\
\hline Escherichia coli & 4 & (8) \\
\hline Haemophilus influenzae & 4 & (8) \\
\hline Enterobacter clocae & 3 & (6) \\
\hline Pseudomonas aeruginosa & 3 & (6) \\
\hline Acinetobacter baumannii & 1 & $(2)$ \\
\hline Enterobacter aerogenes & 1 & $(2)$ \\
\hline Klebsiella oxytoca & 1 & $(2)$ \\
\hline Methicillin-resistant Staphylococcus aureus & 1 & $(2)$ \\
\hline Serratia marcescens & 1 & $(2)$ \\
\hline
\end{tabular}

*For some patients, more than one organism was grown from the sputum sample.

\section{Table 4. Initial Antimicrobial Therapy}

\begin{tabular}{lrc} 
Regimen & No. (\%) of Patients \\
& $(\boldsymbol{n}=\mathbf{4 9 )}$ \\
\hline Ticarcillin-clavulanate & 22 & $(45)$ \\
Ciprofloxacin & 7 & $(14)$ \\
Cefuroxime & 5 & $(10)$ \\
Vancomycin & 5 & $(10)$ \\
Ciprofloxacin + ceftazidime & 2 & $(4)$ \\
Ciprofloxacin + cloxacillin & 2 & $(4)$ \\
Ciprofloxacin + ticarcillin-clavulanate & 2 & $(4)$ \\
Ciprofloxacin + vancomycin & 1 & $(2)$ \\
Ticarcillin-clavulanate + gentamicin & 1 & $(2)$ \\
Ticarcillin-clavulanate + vancomycin & 1 & $(2)$ \\
Vancomycin + ampicillin & 1 & $(2)$ \\
\hline
\end{tabular}

antimicrobials for patients with a low likelihood of pneumonia, as indicated by the CPIS. Analysis of a patient's eligibility for discontinuation of antimicrobials soon after therapy is initiated is a useful exercise, because unnecessary antimicrobial use can lead to significant adverse drug events, including superinfections with multidrug-resistant bacteria. ${ }^{1,10}$ Similar to the day 3 data, by day 7 one-third of the patients were eligible for discontinuation of antimicrobials, yet the drugs were discontinued for only $10 \%$ ( 1 patient out of 10 ). Even if discontinuation on day 3 is perceived as overly aggressive, these data reveal the need for a policy that allows systematic assessment for antimicrobial discontinuation on day 7 .

This study had limitations that must be considered. Most of the data were collected retrospectively, except for chest radiographic data. Retrospective data collection can result in incomplete and inaccurate data sets, which can in turn result in misleading analyses. However, in this study, data entry was complete for all of the necessary variables. The issue of data accuracy is primarily applicable to interpretation of the chest

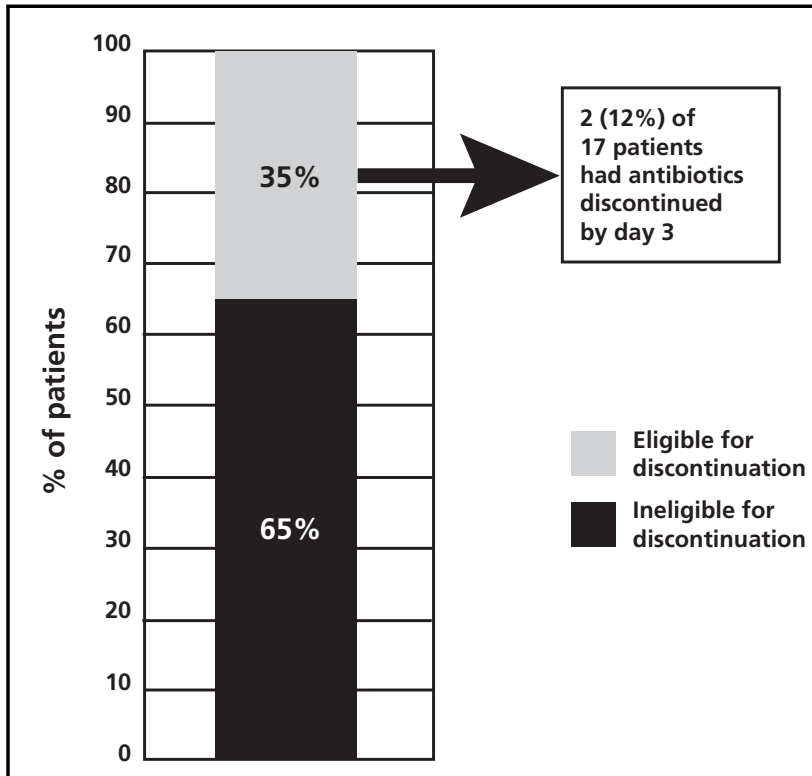

Day 3 assessment $(n=49)$

Figure 2. Eligibility for short-course antimicrobial therapy on day 3.

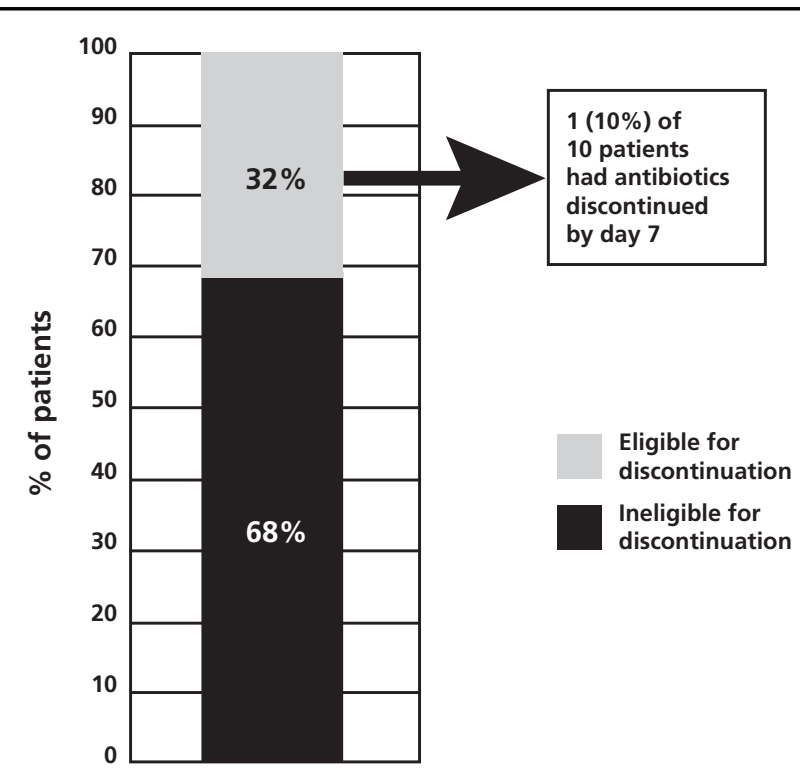

Day 7 assessment $(n=31)$

Figure 3. Eligibility for short-course antimicrobial therapy on day 7 .

radiographs. Efforts were made to obtain this information prospectively from the ICU physician, but this was not always possible for chest radiographs that were obtained on the weekends or after clinical pharmacists' working hours. The fallback was to retrospectively obtain this information from the 
radiologist's formal dictated interpretation, as recorded in the Patient Care Information System. Obtaining this information from 2 sources could have resulted in variability in the radiograph score in the CPIS, which might limit the validity of using CPIS to guide an ICU physician's decision to discontinue antimicrobial therapy for ventilator-associated pneumonia at this institution.

This study was conducted over a 6-month period and therefore had a relatively small sample size. The study sample consisted of a highly selected population of patients with ventilator-associated pneumonia. Patients were excluded from the analysis for numerous reasons. Patients whose pneumonia developed outside of the ICU were excluded because the focus of this study was to gain a full picture of ICU practice in terms of treating this condition. It also was not possible to accurately determine the patient's CPIS on day 0 for this small cohort of patients because of incomplete data. Patients who were deemed to have had a previous episode of ventilator-associated pneumonia less than $48 \mathrm{~h}$ before a new diagnosis of pneumonia were excluded because the new infection might simply have represented a protracted course of the previous episode. Many patients were excluded because of concomitant infections such as intra-abdominal infections and bloodstream infections, which require antimicrobials with a similar spectrum of activity to those required for ventilator-associated pneumonia. These patients were excluded because the duration of antimicrobials might have been influenced by the concomitant infection, rather than the pneumonia, thus confounding the results. Immunocompromised patients such as those who received solid organ transplants were not included because the validity of CPIS for this population has not been determined. Finally, patients were excluded if they were discharged from the ICU or died before day 3 of antimicrobial therapy because these events would have led to incomplete data for the primary end point. Including these patients would have skewed results in favour of shorter-course therapy. Despite the limitations of these strict exclusion criteria, the patients included for analysis generally reflected the overall population of patients admitted to this ICU. For example, the ICU population consists primarily of men in their sixth decade of life with severe illness, as indicated by the APACHE II score on admission. The population analyzed in this study had key differences from the patients in the landmark study that examined use of the CPIS to help determine antimicrobial discontinuation for nosocomial pneumonia. ${ }^{6}$ For example, all of the patients in this study but only $60 \%$ of those in the landmark study ${ }^{6}$ were receiving mechanical ventilation. Also, patients in this study appeared to have a much longer duration of ICU stay than those in the landmark study. ${ }^{6}$

It is not usual practice at the study institution to calculate CPIS for patients with suspected ventilator-associated pneumonia. The ICU clinical pharmacists did not calculate the CPIS, nor were they aware of the CPIS results that were calculated retrospectively by the study pharmacist. Although the patients' CPIS values were not known, knowledge that a study assessing duration of antimicrobial therapy for presumed ventilator-associated pneumonia was being performed might have inadvertently influenced antimicrobial prescribing patterns in the ICU. The hypothesis would be that ICU physicians would make a conscious effort to shorten the treatment course when clinically applicable. However, the results did not reveal this type of bias. In fact, the average duration of therapy (15 days) was unexpectedly high. Also, 90\% of eligible patients received antimicrobials for more than 7 days.

The optimal duration of antimicrobial therapy for ventilatorassociated pneumonia is controversial. The definition of shortcourse antimicrobial therapy for this condition varies from 3 to 8 days, depending on the source. ${ }^{1,8,10,11}$ The primary end point in this study was the more aggressive 3-day definition, although day 7 was also defined a priori as a clinically relevant time to assess for opportunities to discontinue antimicrobial therapy. The findings of this study reveal a significant opportunity to shorten the duration of antimicrobial therapy to either 3 days or 7 days. According to a subgroup analysis from a noninferiority trial comparing 8-day and 15-day antimicrobial courses for ventilator-associated pneumonia, short-course therapy for infections caused by non-lactose-fermenting gram-negative bacilli may not be optimal and appear to predispose patients to more recurrent infections. ${ }^{8}$ More data are required to determine the optimal duration of therapy for these organisms. Recent guidelines for the treatment of ventilator-associated pneumonia in patients with trauma do not base recommendations for duration of therapy on microbiology results and do not recommend predetermined lengths of treatment (e.g., 10, 14 , or 21 days).${ }^{10}$ Instead, the guidelines state that the duration of antimicrobial therapy should be dictated by the patient's clinical response, ${ }^{10}$ which can be facilitated by using the CPIS. Defining treatment duration on the basis of microbiology results may be of little consequence at the current authors' institution, given that non-lactose-fermenting gram-negative bacilli were cultured from only $10 \%$ of patients with ventilatorassociated pneumonia in this study. There is also the concern that shorter-course therapy may not be suitable for patients who do not receive appropriate antimicrobial therapy initially. Patients with ventilator-associated pneumonia who do not receive appropriate initial antimicrobial therapy have significantly higher in-hospital mortality than those whose initial therapy is appropriate. ${ }^{12-15}$ One study revealed that $44 \%$ of patients required modification of the initial antimicrobial regimen because of isolation of resistant microorganisms or lack of clinical response. ${ }^{16}$ Although the definition of appropriateness of initial antimicrobial therapy in the current study was 
based solely on microbiologic information, if available, it is impressive that for more than $95 \%$ of patients with a positive result on sputum culture, the initial antimicrobial therapy was appropriate.

Different versions of the CPIS have been used to aid the diagnosis and treatment of ventilator-associated pneumonia. ${ }^{6,717}$ These different versions have subtleties that may significantly alter the sensitivity and specificity of this score for ventilator-associated pneumonia. The version of the CPIS used in this analysis was identical with the version used in a study of 81 patients who were randomly assigned to receive standard-duration antimicrobial therapy or short-duration (3 days) antimicrobial therapy based on the CPIS. ${ }^{6}$ The reliability of this instrument remains to be confirmed, although one small study revealed good inter-rater reliability (kappa score 0.88) ${ }^{18}$ The CPIS was not used to determine whether the patient did or did not have pneumonia, but rather to identify patients for whom a shorter course of therapy would be acceptable. ${ }^{6}$ It was interesting that fewer than two-thirds of the patients in this study met traditional criteria for the diagnosis of ventilator-associated pneumonia.

The optimal method for diagnosing ventilator-associated pneumonia continues to be a source of great confusion. ${ }^{19}$ The specificity and sensitivity of CPIS relative to bronchoalveolar lavage with quantitative culture for diagnosis of ventilatorassociated pneumonia in patients with trauma has been determined to be unacceptably low. ${ }^{19}$ Patients with trauma made up about one-third of the sample included in this analysis. A small study of patients with burn injury also revealed low sensitivity of CPIS for diagnosis of ventilatorassociated pneumonia. ${ }^{20}$ Based on this evidence, recent guidelines have recommended against using the CPIS to diagnose ventilator-associated pneumonia in patients with trauma. ${ }^{10}$ However, the most recent Canadian guidelines for hospitalassociated pneumonia and ventilator-associated pneumonia incorporate the CPIS into the suggested diagnostic algorithm. ${ }^{21}$ Nevertheless, the authors of the current study do not intend to use CPIS as a diagnostic tool. Rather, serial measurements of CPIS (in conjunction with the clinician's level of suspicion) should serve as a tool to aid decision-making about antimicrobial therapy. The diagnostic method of choice was recently addressed in a multicentre, prospective randomized trial comparing bronchoalveolar lavage with quantitative culture and endotracheal aspiration with nonquantitative culture. The 2 methods produced similar clinical outcomes in terms of 28-day mortality and antimicrobial utilization..$^{22}$ The diagnostic utility of CPIS was not evaluated in that pivotal study.

Another potential limitation of the current study was that the clinician's suspicion of ventilator-associated pneumonia was not evaluated. It can be assumed that there was high suspicion for this condition on day 0 , when antimicrobial therapy was initiated, but the same cannot be assumed for day 3 or day 7 of therapy. For some patients, the physician's clinical suspicion for ventilator-associated pneumonia might have been high on day 3 and day 7 despite a low CPIS value, which might explain the low incidence of antimicrobial discontinuation among eligible patients at these time points. A future antimicrobial discontinuation policy that incorporates CPIS should also account for the clinician's level of suspicion for ventilatorassociated pneumonia.

In summary, a large proportion of critically ill patients who were being treated for ventilator-associated pneumonia at the Vancouver General Hospital were eligible for early antimicrobial discontinuation, at day 3 or day 7 , based on serial calculation of CPIS. However, antimicrobials were discontinued for only a small proportion of these patients. There exists a significant opportunity at the authors' institution to develop and implement an antimicrobial discontinuation policy that uses the CPIS to promote optimal antimicrobial use for ventilator-associated pneumonia.

\section{References}

1. American Thoracic Society; Infectious Diseases Society of America. Guidelines for the management of adults with hospital-acquired, ventilator-associated, and healthcare-associated pneumonia. Am J Respir Crit Care Med 2005;171(4):388-416.

2. Chastre J, Fagon JY. Ventilator-associated pneumonia. Am J Respir Crit Care Med 2002;165(7):867-903.

3. Rello J, Ollendorf DA, Oster G, Vera-Llonch M, Bellm L, Redman R, et al.; VAP Outcomes Scientific Advisory Group. Epidemiology and outcomes of ventilator-associated pneumonia in a large US database. Chest 2002;122(6):2115-2121.

4. NNIS System. National Nosocomial Infections Surveillance (NNIS) System report: data summary from January 1992 through June 2003, issued August 2003. Am J Infect Control 2003;31(8):481-498.

5. Bergmans DC, Bonten MJ, Gaillard CA, van Tiel FH, van der Geest S, de Leeuw PW, et al. Indications for antibiotic use in ICU patients: a one-year prospective surveillance. J Antimicrob Chemother 1997;39(4): 527-535.

6. Singh N, Rogers P, Atwood CW, Wagener MM, Yu VL. Short-course empiric antibiotic therapy for patients with pulmonary infiltrates in the intensive care unit: a proposed solution for indiscriminate antibiotic prescription. Am J Respir Crit Care Med 2000;162(2 Pt 1):505-511.

7. Pugin J, Auckenthaler R, Mili N, Janssens JP, Lew PD, Suter PM. Diagnosis of ventilator-associated pneumonia by bacteriologic analysis of bronchoscopic and nonbronchoscopic "blind" bronchoalveolar lavage fluid. Am Rev Respir Dis 1991;143(5 Pt 1):1121-1129.

8. Chastre J, Wolff M, Fagon JY, Chevret S, Thomas F, Wermert D, et al. Comparison of 8 vs 15 days of antibiotic therapy for ventilator-associated pneumonia in adults: a randomized trial. JAMA 2003;290(19): 2588-2598.

9. Micek ST, Ward S, Fraser VJ, Kollef MH. A randomized controlled trial of an antibiotic discontinuation policy for clinically suspected ventilatorassociated pneumonia. Chest 2004;125(5):1791-1799.

10. Minei JP, Nathens AB, West M, Harbrecht BG, Moore EE, Shapiro MB, et al. Inflammation and the host response to injury, a large-scale collaborative project: patient-oriented research core—standard operating procedures for clinical care. II. Guidelines for prevention, diagnosis and treatment of ventilator-associated pneumonia (VAP) in the trauma patient. J Trauma 2006;60(5):1106-1113.

11. Montravers P, Fagon JY, Chastre J, Lecso M, Dombret MC, Trouillet JL, et al. Follow-up protected specimen brushes to assess treatment in nosocomial pneumonia. Am Rev Respir Dis 1993;147(1):38-44. 
12. Kollef MH, Ward S. The influence of mini-BAL cultures on patient outcomes: implications for the antibiotic management of ventilatorassociated pneumonia. Chest 1998;113(2):412-420.

13. Luna CM, Vujacich P, Niederman MS, Vay C, Gherardi C, Matera J, et al. Impact of BAL data on the therapy and outcome of ventilatorassociated pneumonia. Chest 1997;111(3):676-685.

14. Kollef MH, Sherman G, Ward S, Fraser VJ. Inadequate antimicrobial treatment of infections: a risk factor for hospital mortality among critically ill patients. Chest 1999;115(2):462-474.

15. Iregui M, Ward S, Sherman G, Fraser VJ, Kollef MH. Clinical importance of delays in the initiation of appropriate antibiotic treatment for ventilator-associated pneumonia. Chest 2002;122(1):262-268.

16. Alvarez-Lerma F; ICU-Acquired Pneumonia Study Group. Modification of empiric antibiotic treatment in patients with pneumonia acquired in the intensive care unit. Intensive Care Med 1996;22(5):387-394.

17. Luna CM, Blanzaco D, Niederman MS, Matarucco W, Baredes NC, Desmery $\mathrm{P}$, et al. Resolution of ventilator-associated pneumonia: prospective evaluation of the clinical pulmonary infection score as an early clinical predictor of outcome. Crit Care Med 2003;31(3):676-682.

18. Swoboda SM, Dixon T, Lipsett PA. Can the clinical pulmonary infection score impact ICU antibiotic days? Surg Infect (Larchmt) 2006;7(4): 331-339.

19. Croce MA, Swanson JM, Magnotti LJ, Claridge JA, Weinberg JA, Wood $\mathrm{GC}$, et al. The futility of the clinical pulmonary infection score in trauma patients. J Trauma 2006;60(3):523-528.

20. Pham TN, Neff MJ, Simmons JM et al. The clinical pulmonary infection score poorly predicts pneumonia in patients with burns. J Burn Care Res 2007;28(1):76-79.

21. Rotstein C, Evans G, Born A, Grossman R, Light RB, Magder S, et al. Clinical practice guidelines for hospital-acquired pneumonia and ventilator-associated pneumonia in adults. Can J Infect Dis Med Microbiol 2008;19(1):19-53.

22. Canadian Critical Care Trials Group. A randomized trial of diagnostic techniques for ventilator-associated pneumonia. $N$ Engl J Med 2006; 355(25):2619-2630.

Sean K Gorman, BSC(Pharm), ACPR, PharmD, is a Critical Care Clinical Pharmacotherapeutic Specialist with the Clinical Services Unit - Pharmaceutical Sciences, Vancouver General Hospital, and is a Clinical Associate Professor, Faculty of Pharmaceutical Sciences, University of British Columbia, Vancouver, British Columbia.
Lynne-Michelle M Stewart, BSc(Pharm), ACPR, is a Clinical Pharmacist with the Pharmacy Department, Campbell River and District Hospital, Campbell River, British Columbia.

Richard S Slavik, BSC(Pharm), ACPR, PharmD, FCSHP, is Regional Manager-Professional Practice with the Pharmacy Department, Interior Health Authority, Kelowna, British Columbia, and is a Clinical Associate Professor, Faculty of Pharmaceutical Sciences, University of British Columbia, Vancouver, British Columbia. He is also an associate editor with the CJHP

Jane de Lemos, PharmD, MSc, is Regional Coordinator-Professional Practice with Vancouver Coastal Health - Providence Health Care Regional Shared Pharmacy Services, and Clinical Associate Professor, Faculty of Pharmaceutical Sciences, University of British Columbia, Vancouver, British Columbia.

Dean Chittock, MD, MS, FRCPC, is the Senior Medical Director, Vancouver Coastal Health - Vancouver Acute; Medical Director Intensive Care Unit, Vancouver General Hospital; and Clinica Assistant Professor, Faculty of Medicine, University of British Columbia, Vancouver, British Columbia.

Vinay K Dhingra, MD, FRCPC, is the Medical Manager, Intensive Care Unit, Vancouver General Hospital, and Clinical Assistant Professor, Faculty of Medicine, University of British Columbia, Vancouver, British Columbia.

Juan J Ronco, MD, FRCPC, is a Medical Director, Intensive Care Unit, Vancouver General Hospital, and Clinical Professor, Faculty of Medicine, University of British Columbia, Vancouver, British Columbia.

Harjinder Parwana, BSC(Pharm), ACPR, PharmD, is a Clinical Pharmacotherapeutic Specialist with the Clinical Services Unit Pharmaceutical Sciences, Vancouver General Hospital, Vancouver, British Columbia. At the time this study was performed, she was a Doctor of Pharmacy student in the Faculty of Pharmaceutical Sciences, University of British Columbia.

Address correspondence to:

Dr Sean K Gorman

Clinical Services Unit - Pharmaceutical Sciences

Vancouver General Hospital

855 West 12th Avenue

Vancouver $\mathrm{BC}$

V5Z $1 \mathrm{M} 9$

e-mail: sean.gorman@vch.ca 